\title{
Instrumentation techniques to prevent proximal junctional kyphosis and proximal junctional failure in adult spinal deformity correction-a systematic review of biomechanical studies
}

Citation for published version (APA):

Doodkorte, R. J. P., Vercoulen, T. F. G., Roth, A. K., de Bie, R. A., \& Willems, P. C. (2021). Instrumentation techniques to prevent proximal junctional kyphosis and proximal junctional failure in adult spinal deformity correction-a systematic review of biomechanical studies. The Spine Journal, 21(5), 842854. https://doi.org/10.1016/j.spinee.2021.01.011

Document status and date:

Published: 01/05/2021

DOI:

10.1016/j.spinee.2021.01.011

Document Version:

Publisher's PDF, also known as Version of record

Document license:

Taverne

Please check the document version of this publication:

- A submitted manuscript is the version of the article upon submission and before peer-review. There can be important differences between the submitted version and the official published version of record. People interested in the research are advised to contact the author for the final version of the publication, or visit the DOI to the publisher's website.

- The final author version and the galley proof are versions of the publication after peer review.

- The final published version features the final layout of the paper including the volume, issue and page numbers.

Link to publication

\footnotetext{
General rights rights.

- You may freely distribute the URL identifying the publication in the public portal. please follow below link for the End User Agreement:

www.umlib.nl/taverne-license

Take down policy

If you believe that this document breaches copyright please contact us at:

repository@maastrichtuniversity.nl

providing details and we will investigate your claim.
}

Copyright and moral rights for the publications made accessible in the public portal are retained by the authors and/or other copyright owners and it is a condition of accessing publications that users recognise and abide by the legal requirements associated with these

- Users may download and print one copy of any publication from the public portal for the purpose of private study or research.

- You may not further distribute the material or use it for any profit-making activity or commercial gain

If the publication is distributed under the terms of Article 25fa of the Dutch Copyright Act, indicated by the "Taverne" license above, 


\title{
Instrumentation techniques to prevent proximal junctional kyphosis and proximal junctional failure in adult spinal deformity correction-a systematic review of biomechanical studies
}

\author{
Remco J.P. Doodkorte, MSc ${ }^{\mathrm{a}, *}$, Timon F.G. Vercoulen, MD \\ Alex K. Roth, $\mathrm{PhD}^{\mathrm{a}}$, Rob A. de Bie, $\mathrm{PhD}^{\mathrm{b}}$, Paul C. Willems, MD, $\mathrm{PhD}^{\mathrm{a}}$ \\ ${ }^{a}$ Department of Orthopaedic Surgery, Research School CAPHRI, Maastricht University Medical Center, P. Debyelaan 25, \\ 6229 HX, Maastricht, The Netherlands \\ ${ }^{\mathrm{b}}$ Department of Epidemiology, Research School CAPHRI, Faculty of Health, Medicine and Life Sciences, Maastricht \\ University, P. Debyeplein1, 6229 HA, Maastricht, The Netherlands \\ Received 21 August 2020; revised 8 January 2021; accepted 12 January 2021
}

Abstract

BACKGROUND CONTEXT: Correction of adult spinal deformity (ASD) by long segment instrumented spinal fusion is an increasingly common surgical intervention. However, it is associated with high rates of complications and revision surgery, especially in the elderly patient population. The high construct stiffness of instrumented thoracolumbar spinal fusion has been postulated to lead to a higher incidence of proximal junctional kyphosis (PJK) and failure (PJF). Several cadaveric biomechanical studies have reported on surgical techniques to reduce the incidence of PJF/PJK. As yet, no overview has been made of these biomechanical studies.

PURPOSE: To summarize the evidence of all biomechanical studies that have assessed techniques to reduce PJK/PJF following long segment instrumented spinal fusion in the ASD patient population.

STUDY DESIGN: A systematic review.

METHODS: EMBASE and MEDLINE databases were searched for human and animal cadaveric biomechanical studies investigating the effect of various surgical techniques to reduce PJK/PJF following long segment instrumented thoracolumbar spinal fusion in the adult patient population. Studied techniques, biomechanical test methods, range of motion (ROM), intervertebral disc pressure (IDP) and other relevant outcome parameters were documented.

RESULTS: Twelve studies met the inclusion criteria. Four of these studies included non-human cadaveric material. One study investigated the prophylactic application of cement augmentation (vertebroplasty), whereas the remaining studies investigated semi-rigid junctional fixation techniques to achieve a gradual transition zone of forces at the proximal end of a fusion construct, so-called topping-off. An increased gradual transition zone in terms of ROM compared to pedicle screw constructs was demonstrated for sublaminar tethers, sublaminar tape, pretensioned suture loops, transverse hooks and laminar hooks. Furthermore, reduced IDP was found after the application of sublaminar tethers, suture loops, sublaminar tapes and laminar hooks. Finally, two-level prophylactic vertebroplasty resulted in a lower incidence of vertebral compression fractures in a flexion-compression experiment. CONCLUSIONS: A variety of techniques, involving either posterior semi-rigid junctional fixation or the reinforcement of vertebral bodies, has been biomechanically assessed. However, the low

FDA device/drug status: Approved (Sublaminar tape, Laminar hooks, Cerclage wires, Transverse process hooks, Vertebroplasty). Not approved for this indication (Mersilene tape, Hinged pedicle screws, Tapered rods, Altered rod flexibility, Polycarbonate-urethane rods).

Author disclosures: AKR: Grant: Chemelot InSciTe Project BM2.02 Posture (H, paid directly to institution). PCW: Grant: Chemelot InSciTe Project BM2.02 Posture (H, paid directly to institution). RJPD: Grant: Chemelot InSciTe Project BM2.02 Posture (H, paid directly to institution).
RADB: Grant: Chemelot InSciTe Project BM2.02 Posture (H, paid directly to institution). $\boldsymbol{T F G V}$ : Nothing to disclose.

*Corresponding author. Department of Orthopaedic Surgery, Maastricht University, Universiteitssingel 50, 6229 ER, Maastricht, The Netherlands. Tel.: (31) 43-3881304; fax: (31) 43-3874893.

E-mail address: rjp.doodkorte@maastrichtuniversity.nl (R.J.P. Doodkorte) 
number of studies and variation in study protocols hampers direct comparison of different techniques. Furthermore, determination of what constitutes an optimal gradual transition zone and its translation to clinical practice, would aid comparison and further development of different semirigid junctional fixation techniques. Even though biomechanics are extremely important in the development of PJK/PJF, patient-specific factors should always be taken into account on a caseby-case basis when considering to apply a semi-rigid junctional fixation technique. () 2021 Elsevier Inc. All rights reserved.

Keywords: $\quad$ Adult spinal deformity; Proximal Junctional Kyphosis; Proximal Junctional Failure; Biomechanical; Prophylactic techniques; Systematic review

\section{Introduction}

Over the past decades, instrumented (posterior) spinal fusion for adult spinal deformity (ASD) has become the mainstay of operative treatment. One of the most prevalent complications following surgical correction of ASD is proximal junctional kyphosis (PJK) [1-3]. PJK is generally defined as a postoperative proximal junctional angle increase of $\geq 10^{\circ}$, measured between the inferior end plate of the upper instrumented vertebra (UIV) and the superior end plate of the second supra-adjacent vertebra [4]. The prevalence rates of PJK after ASD surgery vary widely, but have been reported to range between $20 \%$ and $40 \%$ [2,5-7]. Demographic risk factors for the development of PJK include high age, high body mass index, low bone mineral density and the presence of comorbidities [8-11]. Furthermore, surgical risk factors for the development of PJK include a combined anterior and posterior approach, a high magnitude of correction in the sagittal plane and the UIV at T11-L1 [12,13]. PJK does not necessarily have direct clinical implications, but PJK can develop into proximal junctional failure (PJF) due to structural failure (vertebral body fracture, loss of integrity of the posterior ligament complex or vertebral subluxation) with substantial clinical impact. PJF is characterized by mechanical failure, risk of neurological injury, increased deformity, and by the need for revision in up to $47 \%$ of affected patients [14,15].

In 2005, Kim et al. identified construct stiffness as a risk factor for PJK in patients treated by long segment instrumented spinal fusion for adolescent idiopathic scoliosis [1,16]. Ever since, biomechanical studies have evaluated various means of semi-rigid junctional fixation at the proximal end of a rigid construct, also known as topping-off, or the reinforcement of the cranial adjacent vertebrae for PJK and PJF prevention. The evaluated techniques include transverse process hook fixation (TPH), Mersilene tape to reconstruct the posterior ligamentous complex (PLC), and rods with various stiffness. To the authors' knowledge, a systematic review of biomechanically investigated techniques is not available in literature. The objective of this systematic review is to provide an overview of biomechanical studies which assess instrumentation techniques that aim to reduce the incidence of PJK or PJF following long segment instrumented spinal fusion for ASD correction.

\section{Methods}

\section{Study search and selection}

This study was designed and reported according to the preferred reporting items for systematic reviews and metaanalyses guidelines [17]. Electronic databases EMBASE and Medline were searched using the terms in Table 1 on the of May 18, 2020 to identify all relevant articles published from January 2000 onward. No language restrictions were imposed and the reference lists of included articles were manually checked for additional references. Authors were contacted if any of the full-texts, or any of the study data were missing. The articles were de-duplicated in EndNote (X9, Thomson, Reuters, Carlsbad, CA) using the Bramer method [18]. Two authors (RD and TV) individually screened all articles on title and abstract using the Rayyan application for systematic reviews [19].

The identified articles were reviewed based on the titles and abstracts based on the following inclusion criteria: Prophylactic instrumentation techniques assessed to be applied at the proximal end of a posterior thoracolumbar spinal construct ( $>4$ vertebrae), biomechanical studies performed on cadaveric material. Articles were excluded on the following criteria: Clinical studies, finite element modelling studies,

Table 1

EMBASE + MEDLINE 2010 to May 18, 2020 search strategy

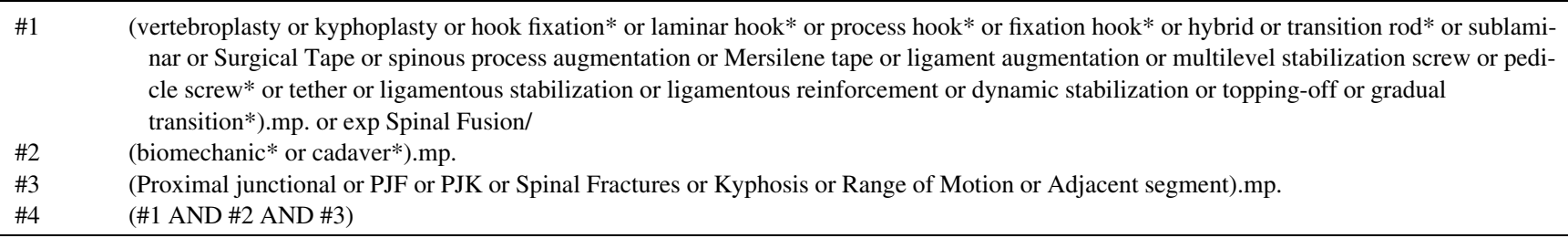


studies solely investigating the cervical or lumbar spine, post-traumatic instrumentation techniques.

\section{Study analysis}

Two independent researchers (RD, TV) extracted the data using a standardized form. The majority of the studies did not report exact range of motions (ROMs) but only graphically represented the results, therefore no meta-analysis was considered. For the interpretation of the data, all statistically significant results were noted. Specific interest was given to the index segment. This is defined as the first intervertebral disc level cephalad to the pedicle screw fixation (Fig. 1, A). Characteristics on the specimen (species, number of specimen, male/female ratio, age, spine segment, index segment, bone mineral density and reasons for exclusions), test setup (the evaluated surgical instrumentation techniques, testing protocol, load limits, test machine and type of measurements) and outcome data (ROMs in; flexion, extension (separate or combined), lateral bending and axial rotation, intervertebral disc pressure (IDP), failure loads, peak loads and fracture levels were registered. Additional comments specific to the assessed techniques were noted.

\section{Quality assessment}

Studies were appraised based on their methodology using the Quality Appraisal for Cadaveric Studies [20], which the authors deemed to be the best fitting risk of bias assessment tool that currently exists for ex vivo biomechanical experiments. This scoring system was developed for observational dissection studies; therefore, the following headings were excluded: "Education of dissecting researchers is stated," "Findings are observed by more than one researcher," "Details about consistency of findings are given," and "Photographs of the observations are included." The remaining 9 of 13 scoring criteria are highly relevant for any cadaveric investigation.

\section{Results}

The search terms identified 2,349 unique articles, and after abstract screening, fifteen articles remained for fulltext screening. Based upon the inclusion criteria, twelve biomechanical studies that investigated techniques to reduce the incidence of PJK/PJF after long segment instrumented posterior spinal instrumentation in the ASD patient population were eligible for inclusion (Fig. 2). Two of the excluded studies assessed fundamental spine biomechanics whereas the other excluded study investigated post-traumatic instrumentation.

\section{Description of included studies}

Eight out of twelve studies used cadaveric human specimen whereas the animal specimen was either porcine [21-23] or calf [24]. Three studies performed flexion-compression testing with varying protocols and did not measure ROMs $[22,25,26]$. The remaining nine studies all measured ROMs, primarily in flexion-extension, of which five studies additionally measured IDP, primarily at the index segment

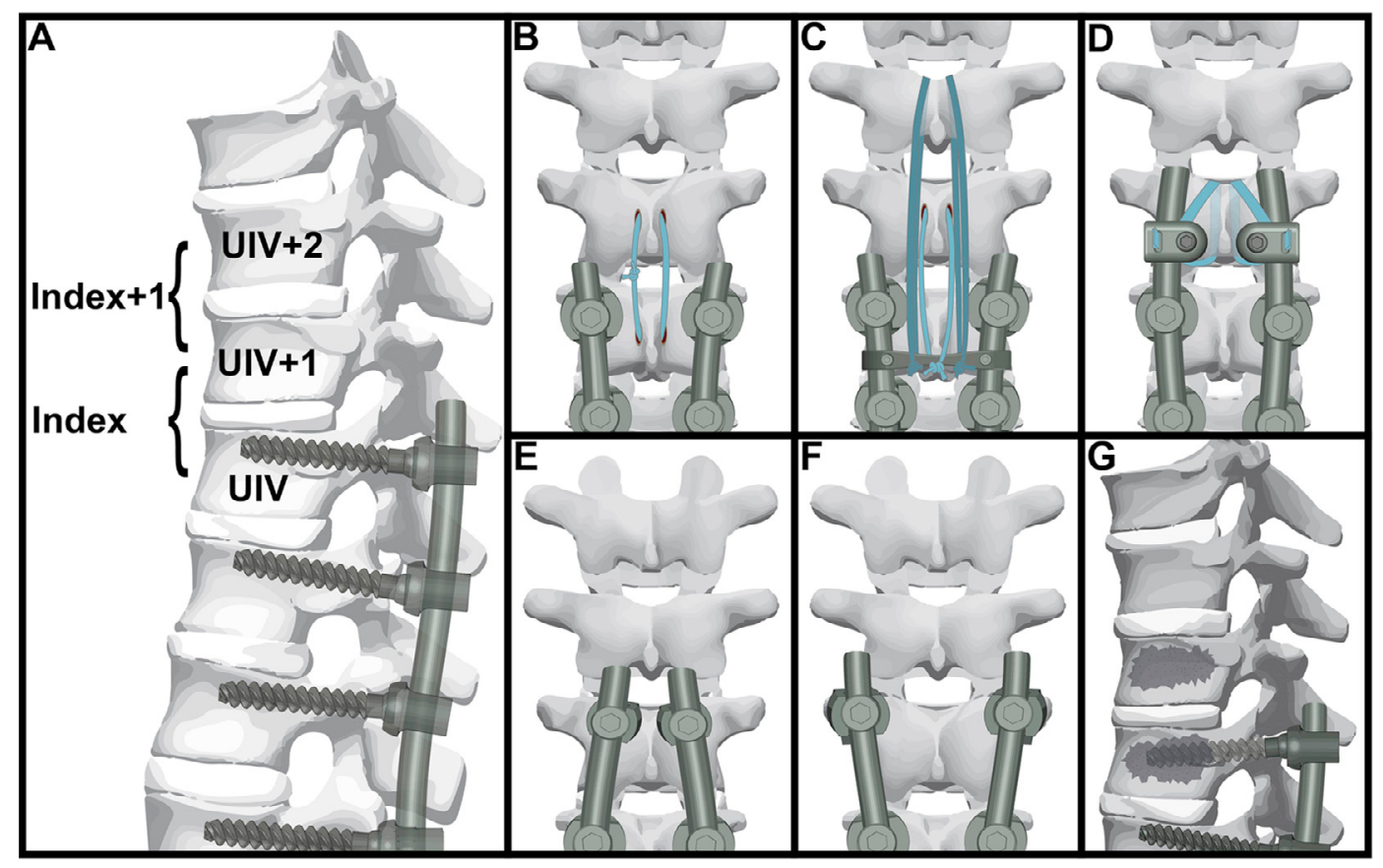

Fig. 1. (A) Throughout this review the most proximal pedicle screw instrumented vertebra is defined as the UIV. Additional vertebrae instrumented as semirigid junctional fixation techniques are called UIV+1 (and UIV+2). Range of motions between UIV +1 and UIV are defined as index segment whereas UIV $+2 / \mathrm{UIV}+1$ is called index+1 segment, (B) Mersilene tape suture loop, (C) Two level tether: UIV+1; spinous process tether (darker shaded blue), UIV+2; sublaminar tethers, (D) sublaminar tape, (E) laminar hooks, (F) transverse process hooks, (G) vertebroplasty. 


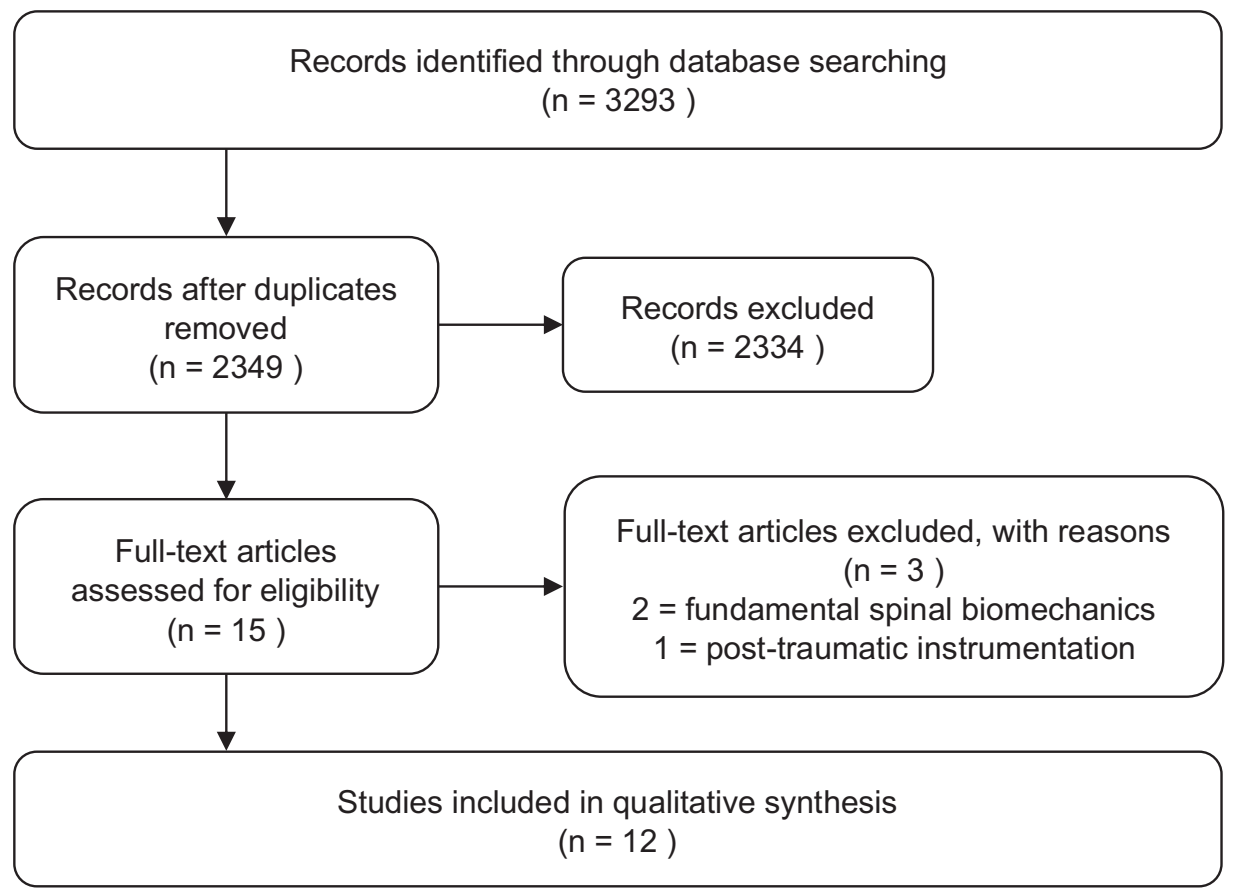

Fig. 2. PRISMA flow diagram of literature search. PRISMA, preferred reporting items for systematic reviews and meta-analyses.

as an approximation of vertebral stresses (Table 2) [21,23,24,27-32].

Four studies investigated the application of suture loops in human cadaveric pure bending experiments [27-30]. Suture loops consist of Mersilene tapes which are passed through the base of multiple consecutive spinous processes (UIV, UIV+1 and sometimes UIV+2) to reinforce the PLC around the index level (Fig. 1, B). Alternatively, three studies investigated the use of tethers in human cadaveric pure bending tests $[27,29,32]$. Tethers were connected from either the spinous process or from the lamina at the UIV+1 (and/or UIV+2) to a crosslink at UIV-1, usually pretensioned by caudal displacement of the crosslink (Fig. 1, C). This was achieved using Mersilene tape [29,32] or commercially available sublaminar wires [27].

Both a human cadaveric study [32], and a calf cadaveric study [24] investigated sublaminar tapes as a semi-rigid junctional fixation system in a pure bending experimental setup. The applied sublaminar tapes were polymeric bands that pass around the lamina of a single vertebra and connect to the ipsilateral spinal rod via a clamp (Fig. 1, D). Additionally, to a one-level application at the index level, Viswanathan et al. also studied sublaminar tapes as a two-level semi-rigid junctional fixation (UIV+1 \& UIV+2) [32]. Beside polymeric sublaminar tapes, Lange et al. also assessed sublaminar cerclage wires at the transitional level [24].

Two different types of hooks have been studied as semirigid junctional fixation. Laminar hooks (LH) at the UIV+1 were studied by Lange et al. in a cadaveric calf experiment and by Metzger et al. in a human cadaveric experiment in which both applied pure bending moments (Fig. 1, E) [24,31]. Transverse process hooks as semi-rigid junctional fixation at UIV+1 were studied in three different studies (Fig. 1, F) $[21,23,26]$. Both Fachinello et al. and Thawrani et al. investigated the use of TPHs in pure bending cadaveric porcine study [21,23]. Mac-Thiong et al. compared TPHs at the junctional zone to pedicle screws in a cadaveric human experiment, in which spine segments were subjected to cyclic flexion-compression until failure [26].

In contrast to the techniques that aim to achieve a gradual transition zone, Kebaish et al. assessed the use of vertebroplasty at the UIV and UIV+1 levels as prophylactic measure to reduced vertebral collapse in a flexion-compression setup till failure [25] (Fig. 1, G).

In addition to the previously described techniques, a number of investigational techniques have been biomechanically assessed. Lange et al. assessed the use of hinged pedicle screws and tapered rods (rods with a smaller diameter at the proximal end) as a means of semi-rigid junctional fixation in the same cadaveric calf study [24]. Besides the TPHs, Facchinello et al. also studied spinal rods with a lower stiffness, either along the complete rod by using a different "superelastic" alloy (Ti-Ni) or only at the transitional area by heat treatment of onlypart of the Ti-Ni rod ("variable stiffness rods") [21]. These rods were studied as stand-alone techniques or and in combination with the TPHs. Finally, Jacobs et al. experimentally assessed the feasibility of using polycarbonate-urethane rods as a means to reduce stresses on the vertebral bodies using porcine cadavers in a flexion-compression setup [22]. 
Table 2

Characteristics of biomechanical studies

\begin{tabular}{|c|c|c|c|c|c|c|}
\hline References & Species (n) & Age & Index segments & Devices & Test protocol & Parameters \\
\hline Kim et al. [28] & Human (8) & $25-76 y$ & $\mathrm{~T} 9-\mathrm{T} 10$ & $\begin{array}{l}\text { Mersilene tape (suture } \\
\text { loop) }\end{array}$ & $8 \mathrm{Nm}(\mathrm{FE}, \mathrm{LB}, \mathrm{AR})$ & ROM \\
\hline Mar et al. (2019a) [30] & Human (9) & $54-85$ y & $\mathrm{T} 11-\mathrm{T} 12$ & $\begin{array}{l}\text { Mersilene tape (suture } \\
\text { loop) }\end{array}$ & $5 \mathrm{Nm}(\mathrm{FE})$ & ROM, IDP (T11-T12), \\
\hline Cho et al. [27] & Human (8) & $25-76 y$ & $\begin{array}{l}\mathrm{T} 9-\mathrm{T} 10 \\
\mathrm{~T} 10-\mathrm{T} 11\end{array}$ & $\begin{array}{l}\text { Mersilene tape (suture } \\
\text { loop) Sublaminar tape } \\
\text { (Tether) }\end{array}$ & $8 \mathrm{Nm}(\mathrm{FE}, \mathrm{LB}, \mathrm{AR})$ & ROM \\
\hline Mar et al. (2019b) [29] & Human (9) & $54-85$ y & $\mathrm{T} 2-\mathrm{T} 3, \mathrm{~T} 3-\mathrm{T} 4$ & $\begin{array}{l}\text { Mersilene tape (tether, 2- } \\
\text { level tether, tether- } \\
\text { suture-loop) }\end{array}$ & $5 \mathrm{Nm}(\mathrm{FE})$ & $\begin{array}{c}\text { ROM, IDP } \\
\text { (T2-T3, } \\
\text { T3-T4) }\end{array}$ \\
\hline Viswanathan et al. [32] & Human (13) & $41-66 y$ & T8-T9, T9-T10 & $\begin{array}{l}\text { Mersilene tape (tether), } \\
\text { Sublaminar tape }\end{array}$ & $6 \mathrm{Nm}(\mathrm{FE}, \mathrm{LB}, \mathrm{AR})$ & $\begin{array}{l}\text { ROM, IDP } \\
\text { (T8-T9, } \\
\text { T9-T10, } \\
\text { T10-T11) }\end{array}$ \\
\hline Lange et al. [24] & Calf (8) & $12-18 \mathrm{~m}$ & L3-L4 & $\begin{array}{l}\text { Sublaminar tape, laminar } \\
\text { hooks, cerclage wires, } \\
\text { hinged pedicle screws, } \\
\text { tapered rods }\end{array}$ & $10 \mathrm{Nm}(\mathrm{FE}, \mathrm{LB}, \mathrm{AR})$ & ROM \\
\hline Metzger et al. [31] & Human (10) & $30-64$ y & $\mathrm{T} 8-\mathrm{T} 9$ & Lamina hooks & $4 \mathrm{Nm}(\mathrm{FE}, \mathrm{LB}, \mathrm{AR})$ & $\begin{array}{r}\text { ROM, IDP } \\
(\mathrm{T} 7-\mathrm{T} 8)\end{array}$ \\
\hline Thawrani et al. [23] & Porcine (13) & $3-4 \mathrm{~m}$ & $\mathrm{~T} 6-\mathrm{T} 7$ & Transverse process hooks & $4 \mathrm{Nm}(\mathrm{FE}, \mathrm{LB})$ & ROM \\
\hline Mac-Thiong et al. [26] & Human $(4 \times 6)$ & $61-85 y$ & $\begin{array}{l}\mathrm{T} 3-\mathrm{T} 4, \mathrm{~T} 7-\mathrm{T} 8, \mathrm{~T} 11-\mathrm{T} 12, \\
\mathrm{~L} 3-\mathrm{L} 4\end{array}$ & Transverse process hooks & Cyclic flexion compression till failure & Failure cycle \\
\hline Facchinello et al. [21] & Porcine (8) & $6-8 \mathrm{~m}$ & L3-L4 & $\begin{array}{l}\text { Transverse process hooks, } \\
\text { Altered rod flexibility }\end{array}$ & $18^{\circ}(\mathrm{FE}, \mathrm{LB})$ & ROM, IDP (L3-L4) \\
\hline Jacobs et al. [22] & Porcine $(4 \times 5)$ & $10-14 \mathrm{~m}$ & L2-L3-L4 & $\begin{array}{l}\text { Polycarbonate-urethane } \\
\text { rods } \\
\text { (65D and } 75 \mathrm{D})\end{array}$ & Cyclic flexion compression & IDP (L2-L3) \\
\hline Kebaish et al. [25] & Human (18) & $26-97$ y & $\begin{array}{l}\text { T10, } \\
\text { T9 \& T10 }\end{array}$ & Vertebroplasty & $\begin{array}{l}\text { Flexion compression } \\
\text { till failure } / 15 \mathrm{~mm}\end{array}$ & Failure load/Fracture ratio \\
\hline
\end{tabular}

IDP, intradiscal pressure (measured at the indicated level); ROM, range of motion; FE, flexion-extension; LB, lateral bending; AR, axial rotation

If test protocol is undefined, the specimen was loaded with pure moments up to the indicated load/rotation angle. 
Table 3

Biomechanical findings of the included studies

\begin{tabular}{|c|c|c|c|c|c|}
\hline References & Condition & $\begin{array}{l}\text { Flexion-extension (flexion, extension or } \\
\text { combined FE measured) }\end{array}$ & Lateral bending (LB) & Torsion (AR) & $\begin{array}{l}\text { F: Additional findings, C: authors } \\
\text { general conclusion }\end{array}$ \\
\hline \multirow[t]{4}{*}{ Cho et al. [27] } & $\begin{array}{l}\text { Sublaminar tether } \\
\quad(250 \mathrm{~N})\end{array}$ & $\begin{array}{l}\text { Reduced flexion ( } 48 \%) \text { and extension ROM. } \\
\text { An increased lordotic angle at the index } \\
\text { segment. }\end{array}$ & - & - & \multirow{4}{*}{$\begin{array}{l}\text { F: 2-level sublaminar tether: A } \\
\text { more gradual transition in rela- } \\
\text { tive motion at each level from } \\
\text { T9-T12 in LB and AR. } \\
\text { C: Polymeric cables can adjust } \\
\text { the ROM as a function of tether } \\
\text { pretension. The authors suggest } \\
\text { load sharing capacity of poste- } \\
\text { rior polymeric cables with the } \\
\text { PLC to avoid excessive strains } \\
\text { and damage to the connective } \\
\text { tissues. }\end{array}$} \\
\hline & $\begin{array}{l}\text { Sublaminar tether } \\
(350 \mathrm{~N})\end{array}$ & $\begin{array}{l}\text { Reduced flexion (57\%), extension ROM. An } \\
\text { increased lordotic angle at the index } \\
\text { segment. }\end{array}$ & Reduced ROM (12\%) & - & \\
\hline & $\begin{array}{l}\text { 2-level Sublaminar } \\
\text { tether }\end{array}$ & $\begin{array}{l}\text { Reduced flexion and extension ROM. An } \\
\text { increased lordotic angle at the index } \\
\text { segment. }\end{array}$ & - & - & \\
\hline & Suture Loop & $\begin{array}{l}\text { An increased lordotic angle at the index } \\
\text { segment. }\end{array}$ & - & - & \\
\hline \multirow[t]{5}{*}{ Facchinello et al. [21],* } & Superelastic rod & $\begin{array}{l}\text { Reduced construct stiffness in flexion, exten- } \\
\text { sion. Increased bending moment on the } \\
\text { pedicle screw in flexion. }\end{array}$ & $\begin{array}{l}\text { Reduced construct } \\
\text { stiffness }\end{array}$ & - & \multirow{5}{*}{$\begin{array}{l}\text { C: A combination of superelastic/ } \\
\text { variable rods with TPH is capa- } \\
\text { ble of providing a smooth tran- } \\
\text { sition between the stabilized } \\
\text { and intact segments. }\end{array}$} \\
\hline & Variable rod & $\begin{array}{l}\text { Reduced construct stiffness in flexion, exten- } \\
\text { sion. Increased bending moment on the } \\
\text { pedicle screw in flexion. }\end{array}$ & $\begin{array}{l}\text { Reduced construct } \\
\text { stiffness }\end{array}$ & - & \\
\hline & TPH & $\begin{array}{l}\text { Increased flexion ROM. Reduced construct } \\
\text { stiffness in flexion, extension. Decreased } \\
\text { forces on upper anchor. }\end{array}$ & $\begin{array}{l}\text { Reduced construct } \\
\text { stiffness }\end{array}$ & - & \\
\hline & $\begin{array}{r}\text { Superelastic } \\
\text { rod + TPH }\end{array}$ & $\begin{array}{l}\text { Increased flexion ROM. Reduced construct } \\
\text { stiffness in flexion, extension. Decreased } \\
\text { forces on upper anchor. }\end{array}$ & $\begin{array}{l}\text { Increased ROM. Reduced } \\
\text { construct stiffness }\end{array}$ & - & \\
\hline & Variable rod $+\mathrm{TPH}$ & $\begin{array}{l}\text { Increased flexion, extension ROM. Reduced } \\
\text { construct stiffness in flexion, extension. } \\
\text { Decreased forces on upper anchor. }\end{array}$ & $\begin{array}{l}\text { Increased ROM. Reduced } \\
\text { construct stiffness }\end{array}$ & - & \\
\hline \multirow[t]{2}{*}{ Jacobs et al. [22] } & $\begin{array}{l}\text { polycarbonate-ure- } \\
\text { thane } 65 \mathrm{D}\end{array}$ & Consistently lower IDP & - & - & \multirow{2}{*}{$\begin{array}{l}\text { C: Lower rod stiffness generates } \\
\text { a lower IDP, more representa- } \\
\text { tive of physical loading. How- } \\
\text { ever, its capability to stabilize } \\
\text { the spine is part of future } \\
\text { studies. }\end{array}$} \\
\hline & $\begin{array}{l}\text { polycarbonate-ure- } \\
\text { thane } 75 \mathrm{D}\end{array}$ & $\begin{array}{l}\text { Consistently lower IDP also compared to } \\
65 \mathrm{D}\end{array}$ & - & - & \\
\hline \multirow[t]{2}{*}{ Kebaish et al. [25] } & UIV vertebroplasty & - & - & - & \multirow[b]{2}{*}{$\begin{array}{l}\text { C: Using vertebroplasty to reduce } \\
\text { the incidence of vertebral com- } \\
\text { pression fractures may be clini- } \\
\text { cally beneficial to prevent PJK } \\
\text { and PJF after long segment } \\
\text { instrumented spinal fusions. }\end{array}$} \\
\hline & $\begin{array}{l}\text { UIV, UIV+1 } \\
\text { vertebroplasty }\end{array}$ & $\begin{array}{l}\text { The number of fractures was significantly } \\
\text { lower compared to both the UIV vertebro- } \\
\text { plasty as well as the nontreated group. }\end{array}$ & - & - & \\
\hline Kim et al. [28] & Suture loop & $\begin{array}{l}\text { An increased lordotic angle at the index } \\
\text { segment. }\end{array}$ & - & - & $\begin{array}{l}\text { C: Suture loops do not prevent } \\
\text { excessive flexion loads on the } \\
\text { proximal junctional segment } \\
\text { following instrumented spinal } \\
\text { fusion. }\end{array}$ \\
\hline
\end{tabular}




\begin{tabular}{|c|c|c|c|c|c|}
\hline References & Condition & $\begin{array}{l}\text { Flexion-extension (flexion, extension or } \\
\text { combined FE measured) }\end{array}$ & Lateral bending (LB) & Torsion (AR) & $\begin{array}{l}\text { F: Additional findings, } \mathrm{C} \text { : authors } \\
\text { general conclusion }\end{array}$ \\
\hline \multirow[t]{5}{*}{ Lange et al. [24] } & Laminar hooks & $\begin{array}{l}\text { FE } 93.2 \% \text { ROM reduction. Increased FE } \\
\text { stiffness to } 1470.6 \%\end{array}$ & $90.0 \%$ ROM reduction & $\begin{array}{l}70.4 \% \text { ROM } \\
\text { reduction }\end{array}$ & \multirow{5}{*}{$\begin{array}{l}\text { C: Laminar hooks, hinged pedi- } \\
\text { cle screws and tapered rods do } \\
\text { not relevantly reduce the stiff- } \\
\text { ness at the end of the hybrid } \\
\text { construct. Cerclage wires and } \\
\text { sublaminar tapes allow gradual } \\
\text { reduction of stiffness at the } \\
\text { proximal end of the long seg- } \\
\text { ment instrumented spinal } \\
\text { fusion. }\end{array}$} \\
\hline & Sublaminar tape & $\begin{array}{l}\text { FE } 58.9 \% \text { ROM reduction. Increased FE } \\
\text { stiffness to } 245.2 \%\end{array}$ & $22.0 \%$ ROM reduction & $\begin{array}{l}22.2 \% \mathrm{ROM} \\
\text { reduction }\end{array}$ & \\
\hline & Cerclage wires & $\begin{array}{l}\text { FE } 57.0 \% \text { ROM reduction. Increased FE } \\
\text { stiffness to } 237.1 \%\end{array}$ & $42.0 \%$ ROM reduction & $\begin{array}{l}44.4 \% \text { ROM } \\
\text { reduction }\end{array}$ & \\
\hline & $\begin{array}{l}\text { Hinged pedicle } \\
\text { screws }\end{array}$ & $\begin{array}{l}\text { FE } 93.2 \% \text { ROM reduction. Increased FE } \\
\text { stiffness to } 816.9 \%\end{array}$ & 93.3\% ROM reduction & $\begin{array}{l}44.4 \% \text { ROM } \\
\text { reduction }\end{array}$ & \\
\hline & Tapered rods & $\begin{array}{l}\text { FE } 86.3 \% \text { ROM reduction. Increased FE } \\
\text { stiffness to } 735.3 \%\end{array}$ & 93.3\% ROM reduction & $\begin{array}{l}44.4 \% \text { ROM } \\
\text { reduction }\end{array}$ & \\
\hline Mac-Thiong et al. [26] & $\begin{array}{l}\text { Transverse process } \\
\text { hooks }\end{array}$ & - & - & - & $\begin{array}{l}\text { C: The study could not demon- } \\
\text { strate a benefit of using TPH } \\
\text { over pedicle screws in an } \\
\text { attempt to decrease the occur- } \\
\text { rence of PJF }\end{array}$ \\
\hline \multirow[t]{5}{*}{ Mar et al. (2019a) [30] } & $\begin{array}{l}\text { Suture loop (no } \\
\text { pretension) }\end{array}$ & $\begin{array}{l}\text { Flexion ROM reduced. This is linearly } \\
\text { related to the amount of pretension. }\end{array}$ & - & - & \multirow{5}{*}{$\begin{array}{l}\text { C: A tether could be used to pre- } \\
\text { serve neutral-zone stiffness and } \\
\text { provides a basis for further } \\
\text { investigation. }\end{array}$} \\
\hline & Suture loop $(22 \mathrm{~N})$ & With increasing pretension, IDPs & - & - & \\
\hline & Suture loop $(44 N)$ & decreased significantly & - & - & \\
\hline & Suture loop $(66 \mathrm{~N})$ & & - & - & \\
\hline & Suture loop $(88 \mathrm{~N})$ & & - & - & \\
\hline \multirow[t]{4}{*}{ Mar et al. (2019b) [29] } & Tether $(22 \mathrm{~N})$ & $\begin{array}{l}\text { Flexion ROM reduced. Reduced IDP at } \\
\text { index and index }+1\end{array}$ & - & - & \multirow{4}{*}{$\begin{array}{l}\text { C: Multiple level tethering can be } \\
\text { biomechanically advantageous } \\
\text { over single level tethering. The } \\
\text { 2-level common method allows } \\
\text { for reduced loading of the } \\
\text { index segment tether loading } \\
\text { and a greater load dissipation to } \\
\text { occur over two adjacent levels. }\end{array}$} \\
\hline & $\begin{array}{l}\text { Tether (2-level } \\
\text { common) }\end{array}$ & $\begin{array}{l}\text { Flexion ROM reduced. Reduced IDP at } \\
\text { index }\end{array}$ & - & - & \\
\hline & $\begin{array}{l}\text { Tether, suture loop (in } \\
\text { series) }\end{array}$ & $\begin{array}{l}\text { Flexion ROM reduced. Reduced IDP at } \\
\text { index }\end{array}$ & - & - & \\
\hline & $\begin{array}{l}\text { Tether (2-level figure } \\
\text { 8) }\end{array}$ & $\begin{array}{l}\text { Flexion ROM reduced. Reduced IDP at } \\
\text { index }\end{array}$ & - & - & \\
\hline \multirow[t]{2}{*}{ Metzger et al. [31] } & Laminar hooks & $\begin{array}{l}\text { Flexion, Extension ROM reduced. More } \\
\text { motion compared to unilateral } \\
\text { hook + unilateral screw. }\end{array}$ & $\begin{array}{l}\text { Reduced ROM. More } \\
\text { motion compared to } \\
\text { unilateral hook+unilat- } \\
\text { eral screw. }\end{array}$ & $\begin{array}{l}\text { Reduced ROM. More } \\
\text { motion compared to } \\
\text { unilateral hook+uni- } \\
\text { lateral screw. }\end{array}$ & \multirow{2}{*}{$\begin{array}{l}\text { C: The study provides a mechani- } \\
\text { cal rationale to consider hooks } \\
\text { at the UIV of a multilevel tho- } \\
\text { racic fusion. Clinical transla- } \\
\text { tion remains necessary. }\end{array}$} \\
\hline & $\begin{array}{l}\text { Laminar hook unilat- } \\
\text { eral, pedicle screw } \\
\text { unilateral }\end{array}$ & Flexion, Extension ROM reduced & Reduced ROM & Reduced ROM. & \\
\hline Thawrani et al. [23],* & $\begin{array}{l}\text { Transverse process } \\
\text { hooks }\end{array}$ & FE ROM reduced. FE stiffness reduced. & - & - & $\begin{array}{l}\text { C: TPH at the UIV provides a } \\
\text { gradual dampening of motion } \\
\text { into the upper segments. }\end{array}$ \\
\hline \multirow[t]{2}{*}{ Viswanathan et al. [32] } & $\begin{array}{l}\text { Sublaminar tape (1- } \\
\text { level) }\end{array}$ & $\begin{array}{l}\text { Reduced flexion ROM by } 55.1 \% \text {. Index IDP } \\
\text { reduced in flexion, extension. }\end{array}$ & Reduced ROM by $23.6 \%$ & $\begin{array}{l}\text { Reduced ROM by } \\
56.7 \% \text {. Index IDP } \\
\text { reduced. }\end{array}$ & \multirow{2}{*}{$\begin{array}{l}\text { F: 2-level sublaminar taping } \\
\text { allows for the most gradual dis- } \\
\text { tribution of forces across the } \\
\text { transitional segments. } \\
\text { C: Compared to tethers, subla- } \\
\text { minar taping yields maximal } \\
\text { motion restriction and reduced }\end{array}$} \\
\hline & $\begin{array}{l}\text { Sublaminar tape (2- } \\
\text { level) }\end{array}$ & $\begin{array}{l}\text { Reduced flexion ROM by } 75.6 \% \text { and exten- } \\
\text { sion ROM to } 78.5 \% \text {. Index IVD reduced in } \\
\text { flexion and extension and in extension at } \\
\text { index }+1 \text {. }\end{array}$ & $\begin{array}{l}\text { Reduced ROM by } 58.4 \% \text {. } \\
\text { Index+1 ROM reduced } \\
\text { by } 21.4 \% . \text { Index IVD } \\
\text { and index }+1 \text { reduced. }\end{array}$ & $\begin{array}{l}\text { Reduced ROM by } \\
65.7 \% \text {. Index }+1 \\
\text { ROM reduced by } \\
51.4 \% \text {. Index IVD }\end{array}$ & \\
\hline
\end{tabular}


The findings of the studies will be described in the "summary of evidence" section clustered as suture loops and tethers, sublaminar tapes, LH, TPHs, vertebroplasty, and investigational implants (Table 3 ).

\section{Critical appraisal of included studies}

The appraisal of methods resulted in an average score of 8.75 of 9 with a minimum score of 8 of 9 for 3 studies. All studies successfully described the following parameters: objective, sample information, condition of the specimens, comprehensive method description, appropriate statistics and comparison of the data to the current evidence. Likewise, all studies scored positive on the precise and thorough presentation of the results. Both Viswanathan et al. and Kim et al. failed to address the limitations of the study $[28,32]$ whereas Mar et al. did not discuss the potential clinical implications of the results [29]. Although not part of the appraisal tool but relevant to mention, Jacobs et al., Kebaish et al., Lange et al., and Mac-Thiong et al. were the only studies that presented numerical results of all conditions $[22,24-26]$.

\section{Summary of evidence}

\section{Suture loops and tethers}

Two studies investigating hand tied (non-tensioned) suture loops, did not find any significant effect of suture loops on the ROM at index level [27,28]. Mar et al. (2019a) investigated the effect of different pretension levels $(0,22$, $44,66,88 \mathrm{~N}$ ) of suture loops on single human functional spinal units (T11-T12) and measured a reduced flexion ROM as well as decreased IDPs with increasing suture loop pretension [30]. In another study by the same authors, these suture loops were pretensioned to 22N [29]. Significant decrease in flexion ROM ( $\mathrm{p}=.007)$ was found at the index segment and IDP levels were found at the index $(\mathrm{p}=.007)$ and index $+1(\mathrm{p}=.002)$ segment.

Mar et al. also compared suture loops to three different tethering configurations tethering from UIV +1 and UIV +2 spinous processes to the crosslink at the UIV [29]. These tethers were likewise pretensioned to $22 \mathrm{~N}$. In this dynamic testing experiment, the pooled data of these two-level tethering techniques resulted in a significantly reduced flexion ROM and IDP at the index segment $(\mathrm{p}=.004, \mathrm{p}<.001)$ but not at index+1 $(\mathrm{p}=.14, \mathrm{p}=.311)$. In the study by Viswanathan et al., using spine segments of eight vertebrae, the sublaminar tethers were pretensioned by distraction via the crosslink and subjected to loading about all three axes of rotation [32]. This experiment resulted in a significantly reduced extension ROM ( $\mathrm{p}=.02)$, but no reduction in flexion ROM at the index segment. Furthermore, a reduced IDP in flexion $(\mathrm{p}=.01)$ and in axial rotation $(\mathrm{p}=.03)$ was found. In turn, these results are similar to the findings of Cho et al. who measured a reduction in flexion $(\mathrm{p}<.05)$ and extension ROM $(\mathrm{p}<.0001)$ in a pretension dependent manner [27]. 
Notably, in this study the tethers were pretensioned to $250 \mathrm{~N}$ and $350 \mathrm{~N}$, which resulted in an altered sagittal alignment towards extension at the index level of $0.7 \pm 0.6^{\circ}$ and $1.0 \pm$ $0.8^{\circ}$, respectively.

\section{Sublaminar tapes}

Applying single level sublaminar tape (as well as sublaminar cerclage wires) as semi-rigid junctional fixation resulted in significantly reduced ROMs at the index segment in flexion-extension $(\mathrm{p}<.001)$ and lateral bending $(\mathrm{p}=.015)$ in a calf spine model [24]. In the study by Viswanathan et al., using human cadaveric spine segments, single level sublaminar banding resulted in significantly reduced flexion, lateral bending and torsion ROM at the index segment, $\mathrm{p}=.01, .003, .003$, respectively [32]. Additionally, a reduced IDP at the index segment was measured in flexion $(\mathrm{p}=.01)$, extension $(\mathrm{p}=.01)$ and torsion $(\mathrm{p}=.02)$.

In the same study, Viswanathan et al. measured the effects of two-level sublaminar tape fixation at the index +1 and index +2 levels on the ROM and IDP. Compared to pedicle fixation, this resulted in a large significant reduction of ROMs at the index segment (flexion, $\mathrm{p}=.02$, extension, $\mathrm{p}=.04$, lateral bending, $\mathrm{p}=.02$, torsion, $\mathrm{p}=.02$ ) and a significantly reduced ROM in lateral bending and torsion at UIV $+2(\mathrm{p}=.03, \mathrm{p}=.02)$. Furthermore, the IDP was reduced in extension $(\mathrm{p}=.05)$, lateral bending $(\mathrm{p}=.05)$ and torsion $(\mathrm{p}=.04)$ at the index +1 level in addition to all directions at the index segment (flexion, $\mathrm{p}=.02$, extension, $\mathrm{p}=.02$, lateral bending, $\mathrm{p}=.05$, torsion, $\mathrm{p}=.05$ ).

\section{Laminar hooks}

Lange et al. found a ROM reduction of $93.2 \%(\mathrm{p}<.001)$, $90.0 \%(\mathrm{p}<.001)$ and $70.4 \%(\mathrm{p}<.001)$ in flexion-extension, lateral bending and torsion respectively, when introducing $\mathrm{LH}$ as a semi-rigid junctional fixation in a cadaveric calf experiment [24]. Therefore, the authors concluded that this fixation technique results in a stiff fixation analogous to pedicle screw fixation. In contrast, Metzger et al. showed significantly higher ROMs in lateral bending $(\mathrm{p}<.05)$ and torsion $(\mathrm{p}<.05)$ and a lower stiffness in torsion $(\mathrm{p}<.05)$ at the index segment when replacing pedicle screws with $\mathrm{LH}$ in a human cadaveric experiment. Additionally, the IDP at the index +1 level was significantly reduced in flexion $(\mathrm{p}<.001)$ and extension $(\mathrm{p}<.001)$ [31].

\section{Transverse process hooks}

Comparing the TPH semi-rigid junctional fixation technique to an all-pedicle screw construct in a human cadaveric cyclic flexion-compression experiment, resulted in similar number of cycles until failure $(\mathrm{p}=.9)$ [26]. Both Facchinello et al. and Thawrani et al. compared an all-pedicle screw construct to a construct that replaces the most proximal pedicle screws with TPH [21,23]. Therefore, the comparison is slightly different than studies that apply techniques as an additional level of instrumentation. Facchinello et al. measured a significantly higher flexion ROM $(\mathrm{p}<.05)$ and significantly lower overall construct stiffness in flexion and extension (both $\mathrm{p}<.05$ ) at the index segment when applying TPH [21]. Thawrani et al. measured an increased flexion-extension $(\mathrm{p}=.008)$ and lateral bending ROM $(\mathrm{p}<.05)$ at the index segment [23]. Alternatively, calculated, the TPH reduced the stiffness by $>50 \%$ compared to an all-pedicle screw construct.

\section{Prophylactic vertebroplasty}

Vertebroplasty was tested as a one-level and a two-level prophylactic measure to reduce PJF [25]. Vertebroplasty was experimentally assessed by $15 \mathrm{~mm}$ of flexion compression on T7-L5 segments instrumented with pedicle fixation from T10 to L5. Although no difference in peak loads till failure was measured $(\mathrm{p}=.62)$, the 2-level vertebroplasty group had significantly less vertebral fractures compared to the 1-level vertebroplasty, and the non-augmented group $(\mathrm{p}=.021)$.

\section{Investigational techniques}

A number of investigational (noncommercially available) techniques that aimed to achieve gradual-junctional ROM transition have been identified. Most of these techniques altered the mechanical characteristics of the spinal instrumentation rods and one study investigated the use of hinged pedicle screws $[21,22,24]$. Both hinged pedicle screws and tapered rods were studied by Lange et al. in the same study mentioned previously, however, neither technique yielded any clinically relevant effect [24].

Besides TPH, Facchinello et al. investigated the use of "superelastic" rods and "variable stiffness" rods [21]. Utilizing the spinal rods as stand-alone semi-rigid junctional fixation technique did not result in significant effects on ROM at the index segments.

Finally, polycarbonate-urethane rods were experimentally compared to titanium rods in a flexion-compression setup [22]. This did result in significantly reduced IDPs at the index segment for both type of polycarbonate-urethane rods (Shore hardness 65D $\mathrm{p}<.05$, Shore hardness 75D $\mathrm{p}<.05)$. Unfortunately, data regarding other levels including ROM data was not available.

\section{Discussion}

This systematic review outlines the current literature regarding biomechanically tested devices to prevent PJK and PJF following long segment instrumented spinal fusion for ASD. A total of twelve biomechanical studies were identified that assessed the effect of semi-rigid junctional fixation proximal to the pedicle screw fixation or reinforcement and/or augmentation of the vertebral bodies.

\section{Biomechanical evidence}

Suture loops and tethers have been shown to significantly reduce the flexion and/or extension ROM. However, these effects appear only to be biomechanically relevant if 
sufficient pretension is applied while the effect-size was also shown to depend on the amount of pretension [27,29,30,32]. Overall, reduced flexion-extension ROMs were accompanied with decreased IDPs, indicating reduced transfer of stresses to the vertebral bodies, and therefore the possible reduction of both PJK and PJF incidence. Furthermore, pretensioning the tether to 250 and $350 \mathrm{~N}$ resulted in an altered sagittal alignment towards extension at the index level, with a transfer of loads to the posterior elements. On the one hand, this could be interpreted as either an additional measure of correction, on the other it could also result in increased posterior stresses at proximal levels and elements prone to failure. Cho et al. also subjected the spine segments to lateral bending and torsion but only found significant reduction in lateral bending ROM only with $350 \mathrm{~N}$ pretensioned tethers [27]. The limited demonstration of effectiveness in lateral bending and torsion may be due to the fact these techniques are applied in the sagittal plane and thus lack a moment arm to affect the ROM and stiffness in these directions.

Sublaminar taping has been applied as a one- or twolevel semi-rigid junctional fixation technique [24,32]. Both one- and two-level techniques demonstrated reduced ROMs and IDP at the index segment in all three principal axes of movement. Additionally, sublaminar tape as a two level semi-rigid junctional fixation technique yielded a larger reduction of ROM at the index segment and reduced ROM at the index +1 level. The authors claim to attain a gradual transition in this setup, but the flexion-extension ROM at the index level is limited to $20 \%$ to $25 \%$ of the native condition, and as derived from the graphs, not significantly different from the index-1 segment. This could be considered similar to pedicle screw fusion and thus not a semi-rigid fixation. The findings in these two studies suggest that sublaminar taping is a feasible technique. However, the lack of a definition of an optimal gradual junctional transition hampers the predictability of clinical effectiveness.

Two studies have compared the use of LH to pedicle screw fixation $[24,31]$. The study performed with human cadaveric specimens demonstrated a beneficial effect on a gradual junctional transition, whereas the study on calf spines did not. The effectiveness of hook fixation is dependent on the anatomy of the vertebrae and thus may explain the differences between these studies. Although Metzger et al. demonstrated a significant difference in ROM, the small differences in observed IDP might suggest limited effectiveness in clinical practice compared to other techniques [31]. The use of TPH demonstrated promising quasistatic biomechanical characteristics in two animal cadaveric studies but not in a human cadaveric study. In the human study, the specimens were subjected to cyclic flexion-compression, however, the authors themselves debate whether or not this regime would be able to discriminate between different techniques. In both studies with animal spines, the TPH were considered as a replacement of pedicle screws instead of and addition to a pedicle screw construct, which could have obscured results. Nevertheless, TPH showed a more gradual transition in ROM compared to a pedicle screw construct. Similar to the laminar hook fixation, the anatomical differences between animal and human vertebrae limit extrapolation of the results to the human clinical application.

Previously, Hohn et al. quantified the bone mineral density of individual regions of lumbar vertebrae and showed that laminar bone mineral density was overall higher compared to the density of the pedicles, whereas in the transverse processes bone mineral density was lower [33]. Furthermore, it has been demonstrated that resistance to failure in laminar hook and sublaminar wire fixation is not correlated to overall bone mineral density, whereas a negative correlation between resistance to failure and overall bone mineral density has been demonstrated for spinous process wiring and pedicle screw fixation [34]. BMD-independent pull-out resistance and the potential to introduce a gradual ROM transition at the junctional zone provide a clear rationale for the use of (sub)-laminar fixation at the terminal ends of spinal constructs in the often osteopenic ASD patient population.

Kebaish et al. studied the effect of prophylactic vertebroplasty of the UIV and UIV+1 vertebrae as a technique to reduce the risk of PJF [25]. Although a frequency difference in vertebral fractures was measured, the applied loading regime of $15 \mathrm{~mm}$ displacement is of arbitrary nature and thus hampers extrapolation of the findings to a potential clinical implication. In a similar study by Higgins et al. individual vertebrae were biomechanically assessed on the effectiveness of prophylactic vertebroplasty for osteoporotic patients [35]. They concluded that the introduction of $20 \%$ bone cement could increase failure strength, especially in the vertebrae with a lower BMD. However, the effects may be limited to the lower thoracic and lumbar spine. So even though this systematic review did not yield wide biomechanical evidence for the prophylactic application of vertebroplasty, its application in the ASD population with a reduced $\mathrm{BMD}$ has a clear rationale. Unfortunately, as vertebroplasty does not affect the ROM of spine segments, comparison to other techniques that do affect the biomechanical behavior in non-failure loading regimes is cumbersome.

The use of polycarbonate-urethane rods requires further laboratory studies on the ROM effects whereas hinged pedicle screws, and variable stiffness metal rods did not yield any clinically relevant differences.

\section{Clinical relevance}

It must be noted that clinical translation of biomechanical findings can be cumbersome, yet, some of the techniques have been applied in clinical studies involving the patient population with a degenerative spine.

The pretension dependent effectiveness of suture loops and tethers which has been demonstrated biomechanically, has been shown clinically as well. In a clinical pilot study 
by Buell et al., patients instrumented with suture loops as a semi-rigid junctional fixation technique had significantly lower PJK incidence compared to patients without semirigid junctional fixation [36]. Moreover, this difference was more prominent in patients treated with pretensioned tethers fixed via a crosslink compared to hand tied suture loops. Authors suggest this was likely due to the pretension applied by distal distraction of the crosslink after knotting the polymer tape. A clinical safety study by Viswanathan et al. assessed the use of single level sublaminar tape in an ASD patient population and demonstrated non-inferiority. A reduced incidence of PJK and or PJF was not demonstrated, but the study setup was not sufficiently powered to show a potential effect [37].

Although both laminar- and TPH fixation have been biomechanically assessed, clinical evaluation of this concept has only been performed for TPH. In a retrospective cohort study by Hassanzadeh et al. a lower incidence of PJK and a lower need for revision for PJK was found. However, differences in follow-up length and initial patient characteristics may have skewed these results [38].

Two level prophylactic vertebroplasty was only assessed in one biomechanical study, but clinically this concept has been clinically assessed in multiple prospective and retrospective cohort studies [39-43]. The beneficial clinical outcomes for the ASD patient population treated with prophylactic vertebroplasty support this treatment.

\section{Strengths and limitations}

The current study provides a thorough overview of the spinal instrumentation techniques that were biomechanically assessed to prevent PJK and PJF. The twelve identified studies vary in terms of instrumentation technique and the subjected mechanical loading regime. Not only has this hampered direct comparison of the different techniques, but also the limited numerical presentation of the results further complicated interpretation of the results. The majority of the authors have chosen to graphically represent results and limited description of numerical outcomes to the significant findings. Moreover, biomechanical literature lacks a clear definition of an optimal gradual transition zone. Therefore, this study recommends more thorough numerical presentation of biomechanical findings and the authors want to express the need for a description of the ideal transition zone by comparative evaluation of thorough biomechanical studies and clinical efficacy studies.

A number of authors argue the loss of integrity of the PLC as a risk for the development of PJK and PJF, whereas a shifted load towards anterior, or a weakened integrity of the vertebral body could well be the underlying process in developing PJK. Therefore, insights into the stress and strain distributions of all structures in the uninstrumented, as well as instrumented, degenerative spine could provide further insight in the relation between biomechanical findings and clinical relevance, and help find a rationale for the ideal technique to reduce the incidence of PJF. Another important biomechanical characteristic of the spine is the neutral zone. Already reported in 1992 by Panjabi [44], the neutral zone provides information on the stabilization of the spine. The majority of the studies measured ROM and/ or IDP. Strikingly, only one article mentioned the measurement of the neutral zone, and none reported about it [32]. Nevertheless, the neutral zone could provide further insight in the stresses and strains within the spine segments.

In an effort to methodologically assess the included studies, an adaptation of the Quality Appraisal for Cadaveric Studies scoring was employed [20]. All studies achieved a score of eight or nine out of nine, implicating methodologically sound studies. However, the scoring system was unable to discriminate between the clinical relevance of various loading regimes, comparability of the results to other studies and presentation of non-significant findings. Hence, we suggest the development of a new scoring system specifically for the methodological assessment of biomechanical studies.

\section{Conclusion}

This systematic review provides an overview of the ex vivo biomechanical evidence for the wide variety of surgical techniques assessed to reduce the incidence of PJK and PJF after long segment instrumented spinal fusion in the ASD population. Due to variation in the methodological execution of the experiments, the assessed instrumentation techniques studies could not be directly compared. However, it is clear that the application of polymeric cable systems, either as tether in the sagittal plane or via bilateral sublaminar fixation, shows great potential. Both groups of techniques demonstrated reduced ROM and IDP at the transition zone in flexion-extension with additional effects in lateroflexion and torsion for sublaminar tapes. Notably, preload the tethers and tapes using a tensioning device and/or caudal displacement of the tether fixated crosslink is a prerequisite in order to be effective. Both laminar and TPHs also appear to be an effective measure to introduce a gradual ROM transition in the junctional zone. As an alternative to semi-rigid junctional fixation, prophylactic vertebroplasty may be an effective technique to reduce the incidence of PJF however, the biomechanical evidence was meager. Moreover, the nature of the technique being applied as a reinforcement of the bony structures instead of altering the dynamic characteristics of spine segments makes ex vivo comparison to the other techniques evaluated in this systematic review cumbersome. Finally, altered rod stiffness' and hinged pedicle screws have been subject of biomechanical experiments, but neither yield clinically relevant results.

Systematic side-by-side comparisons in pure-moment loaded human cadaveric spines could further aid development and refinement of prophylactic techniques to reduce the incidence of PJK and PJF after long segment 
instrumented spinal fusion of ASD patients. Furthermore, determination of what constitutes an optimal gradual transition zone and its translation to clinical practice, would aid comparison and further development of different semi-rigid junctional fixation techniques. Even though biomechanics are extremely important in the development of PJK/PJF, patient-specific factors should always be taken into account on a case-by-case basis when considering to apply a semirigid junctional fixation technique.

\section{Acknowledgment}

This research was performed within the Chemelot InSciTe framework and the authors have no financial or competing interests related to this work.

\section{References}

[1] Kim YJ, Bridwell KH, Lenke LG, Kim J, Cho SK. Proximal junctional kyphosis in adolescent idiopathic scoliosis following segmental posterior spinal instrumentation and fusion: minimum 5-year followup. Spine (Phila Pa 1976) 2005;30(18):2045-50.

[2] Kim HJ, Lenke LG, Shaffrey CI, Van Alstyne EM, Skelly AC. Proximal junctional kyphosis as a distinct form of adjacent segment pathology after spinal deformity surgery: a systematic review. Spine (Phila Pa 1976) 2012;37(Suppl. 22):S144-64.

[3] Faundez AA, Richards J, Maxy P, Price R, Leglise A, Le Huec JC. The mechanism in junctional failure of thoraco-lumbar fusions. Part II: analysis of a series of PJK after thoraco-lumbar fusion to determine parameters allowing to predict the risk of junctional breakdown. Eur Spine J 2018;27(Suppl. 1):139-48.

[4] Glattes RC, Bridwell KH, Lenke LG, Kim YJ, Rinella A, Edwards 2nd C. Proximal junctional kyphosis in adult spinal deformity following long instrumented posterior spinal fusion: incidence, outcomes, and risk factor analysis. Spine (Phila Pa 1976) 2005;30(14):1643-9.

[5] Diebo BG, Shah NV, Stroud SG, Paulino CB, Schwab FJ, Lafage V. Realignment surgery in adult spinal deformity: prevalence and risk factors for proximal junctional kyphosis. Orthopade 2018;47(4):301-9.

[6] Lau D, Clark AJ, Scheer JK, Daubs MD, Coe JD, Paonessa KJ, et al. SRS Adult Spinal Deformity Committee. Proximal junctional kyphosis and failure after spinal deformity surgery: a systematic review of the literature as a background to classification development. Spine (Phila Pa 1976) 2014;39(25):2093-102.

[7] Hyun SJ, Lee BH, Park JH, Kim KJ, Jahng TA, Kim HJ. Proximal junctional kyphosis and proximal junctional failure following adult spinal deformity surgery. Korean J Spine 2017;14(4):126-32.

[8] Liu FY, Wang T, Yang SD, Wang H, Yang DL, Ding WY. Incidence and risk factors for proximal junctional kyphosis: a meta-analysis. Eur Spine J 2016;25(8):2376-83.

[9] O'Leary PT, Bridwell KH, Lenke LG, Good CR, Pichelmann MA, Buchowski JM, et al. Risk factors and outcomes for catastrophic failures at the top of long pedicle screw constructs: a matched cohort analysis performed at a single center. Spine (Phila Pa 1976) 2009;34 (20):2134-9.

[10] Kim DK, Kim JY, Kim DY, Rhim SC, Yoon SH. Risk factors of proximal junctional kyphosis after multilevel fusion surgery: more than 2 years follow-up data. J Korean Neurosurg Soc 2017;60 (2):174-80.

[11] Diebo BG, Jalai CM, Challier V, Marascalchi BJ, Horn SR, Poorman $\mathrm{GW}$, et al. novel index to quantify the risk of surgery in the setting of adult spinal deformity: a study on 10,912 patients from the nationwide inpatient sample. Clin Spine Surg 2017;30(7):E993-E9.
[12] Park SJ, Lee CS, Chung SS, Lee JY, Kang SS, Park SH. Different risk factors of proximal junctional kyphosis and proximal junctional failure following long instrumented fusion to the sacrum for adult spinal deformity: survivorship analysis of 160 patients. Neurosurgery 2017;80(2):279-86.

[13] Kim HJ, Bridwell KH, Lenke LG, Park MS, Song KS, Piyaskulkaew $\mathrm{C}$, et al. Patients with proximal junctional kyphosis requiring revision surgery have higher postoperative lumbar lordosis and larger sagittal balance corrections. Spine (Phila Pa 1976) 2014;39(9):E576-80.

[14] Hart RA, McCarthy I, Ames CP, Shaffrey CI, Hamilton DK, Hostin R. Proximal junctional kyphosis and proximal junctional failure. Neurosurg Clin N Am 2013;24(2):213-8.

[15] Hart R, McCarthy I, O'Brien M, Bess S, Line B, Adjei OB, et al. International Spine Study Group. Identification of decision criteria for revision surgery among patients with proximal junctional failure after surgical treatment of spinal deformity. Spine (Phila Pa 1976) 2013;38(19):E1223-7.

[16] Kim YJ, Lenke LG, Bridwell KH, Kim J, Cho SK, Cheh G, et al. Proximal junctional kyphosis in adolescent idiopathic scoliosis after 3 different types of posterior segmental spinal instrumentation and fusions: incidence and risk factor analysis of 410 cases. Spine (Phila Pa 1976) 2007;32(24):2731-8.

[17] Moher D, Liberati A, Tetzlaff J, Altman DG, Group P. Preferred reporting items for systematic reviews and meta-analyses: the PRISMA statement. PLoS Med 2009;6(7):e1000097.

[18] Bramer WM, Giustini D, de Jonge GB, Holland L, Bekhuis T. Deduplication of database search results for systematic reviews in EndNote. J Med Libr Assoc 2016;104(3):240-3.

[19] Ouzzani M, Hammady H, Fedorowicz Z, Elmagarmid A. Rayyan-a web and mobile app for systematic reviews. Syst Rev 2016;5(1):210.

[20] Wilke J, Krause F, Niederer D, Engeroff T, Nurnberger F, Vogt L, et al. Appraising the methodological quality of cadaveric studies: validation of the QUACS scale. J Anat 2015;226(5):440-6.

[21] Facchinello Y, Brailovski V, Petit Y, Brummund M, Tremblay J, Mac-Thiong JM. Biomechanical assessment of the stabilization capacity of monolithic spinal rods with different flexural stiffness and anchoring arrangement. Clin Biomech (Bristol, Avon) 2015;30 (10): $1026-35$.

[22] Jacobs E, Roth AK, Arts JJ, van Rhijn LW, Willems PC. Reduction of intradiscal pressure by the use of polycarbonate-urethane rods as compared to titanium rods in posterior thoracolumbar spinal fixation. J Mater Sci Mater Med 2017;28(10):148.

[23] Thawrani DP, Glos DL, Coombs MT, Bylski-Austrow DI, Sturm PF. Transverse process hooks at upper instrumented vertebra provide more gradual motion transition than pedicle screws. Spine (Phila Pa 1976) 2014;39(14):E826-32.

[24] Lange T, Schmoelz W, Gosheger G, Eichinger M, Heinrichs CH, Boevingloh AS, et al. Is a gradual reduction of stiffness on top of posterior instrumentation possible with a suitable proximal implant? A biomechanical study. Spine J 2017;17(8):1148-55.

[25] Kebaish KM, Martin CT, O'Brien JR, LaMotta IE, Voros GD, Belkoff SM. Use of vertebroplasty to prevent proximal junctional fractures in adult deformity surgery: a biomechanical cadaveric study. Spine J 2013;13(12):1897-903.

[26] Mac-Thiong JM, Levasseur A, Parent S, Petit Y. The influence of proximal anchors on the risk of proximal junctional fracture in the osteoporotic spine: biomechanical comparison between pedicle screws and transverse process hooks. J Spinal Disord Tech 2014;27 (2):E49-54.

[27] Cho SK, Caridi J, Kim JS, Cheung ZB, Gandhi A, Inzana J. Attenuation of proximal junctional kyphosis using sublaminar polyester tension bands: a biomechanical study. World Neurosurg 2018;120: e1136-e42.

[28] Kim JS, Cheung ZB, Arvind V, Caridi J, Cho SK. Role of posterior ligamentous reinforcement in proximal junctional kyphosis: a cadaveric biomechanical study. Asian Spine J 2019;13(1):68-76. 
[29] Mar DE, Burton DC, McIff TE. Biomechanics of prophylactic tethering for proximal junctional kyphosis: comparison of posterior tether looping techniques. Spine Deform 2019;7(2):197-202.

[30] Mar DE, Clary SJ, Burton DC, McIff TE. Biomechanics of prophylactic tethering for proximal junctional kyphosis: characterization of spinous process tether pretensioning and pull-out force. Spine Deform 2019;7(2):191-6.

[31] Metzger MF, Robinson ST, Svet MT, Liu JC, Acosta FL. Biomechanical analysis of the proximal adjacent segment after multilevel instrumentation of the thoracic spine: do hooks ease the transition? Global Spine J 2016;6(4):335-43.

[32] Viswanathan VK, Ganguly R, Minnema AJ, DeVries Watson NA, Grosland NM, Fredericks DC, et al. Biomechanical assessment of proximal junctional semi-rigid fixation in long-segment thoracolumbar constructs. J Neurosurg Spine 2018;30(2):184-92.

[33] Hohn EA, Chu B, Martin A, Yu E, Telles C, Leasure J, et al. The pedicles are not the densest regions of the lumbar vertebrae: implications for bone quality assessment and surgical treatment strategy. Global Spine J 2017;7(6):567-71.

[34] Coe JD, Warden KE, Herzig MA, McAfee PC. Influence of bone mineral density on the fixation of thoracolumbar implants. A comparative study of transpedicular screws, laminar hooks, and spinous process wires. Spine (Phila Pa 1976) 1990;15(9):902-7.

[35] Higgins KB, Harten RD, Langrana NA, Reiter MF. Biomechanical effects of unipedicular vertebroplasty on intact vertebrae. Spine (Phila Pa 1976) 2003;28(14):1540-7. discussion 8.

[36] Buell TJ, Buchholz AL, Quinn JC, Bess S, Line BG, Ames CP, et al. A pilot study on posterior polyethylene tethers to prevent proximal junctional kyphosis after multilevel spinal instrumentation for adult spinal deformity. Oper Neurosurg (Hagerstown) 2019;16(2):256-66.

[37] Viswanathan VK, Kukreja S, Minnema AJ, Farhadi HF. Prospective assessment of the safety and early outcomes of sublaminar band placement for the prevention of proximal junctional kyphosis. J Neurosurg Spine 2018;28(5):520-31.
[38] Hassanzadeh H, Gupta S, Jain A, El Dafrawy MH, Skolasky RL, Kebaish KM. Type of anchor at the proximal fusion level has a significant effect on the incidence of proximal junctional kyphosis and outcome in adults after long posterior spinal fusion. Spine Deform 2013;1(4):299-305.

[39] Ghobrial GM, Eichberg DG, Kolcun JPG, Madhaven K, Lebwohl $\mathrm{NH}$, Green BA, et al. Prophylactic vertebral cement augmentation at the uppermost instrumented vertebra and rostral adjacent vertebra for the prevention of proximal junctional kyphosis and failure following long-segment fusion for adult spinal deformity. Spine J 2017;17 (10):1499-505.

[40] Han S, Hyun SJ, Kim KJ, Jahng TA, Jeon SI, Wui SH, et al. Effect of vertebroplasty at the upper instrumented vertebra and upper instrumented vertebra +1 for prevention of proximal junctional failure in adult spinal deformity surgery: a comparative matched-cohort study. World Neurosurg 2019;124:e436-44.

[41] Theologis AA, Burch S. Prevention of acute proximal junctional fractures after long thoracolumbar posterior fusions for adult spinal deformity using 2-level cement augmentation at the upper instrumented vertebra and the vertebra 1 level proximal to the upper instrumented vertebra. Spine (Phila Pa 1976) 2015;40 (19):1516-26.

[42] Raman T, Miller E, Martin CT, Kebaish KM. The effect of prophylactic vertebroplasty on the incidence of proximal junctional kyphosis and proximal junctional failure following posterior spinal fusion in adult spinal deformity: a 5-year follow-up study. Spine J 2017;17 (10):1489-98

[43] Martin CT, Skolasky RL, Mohamed AS, Kebaish KM. Preliminary results of the effect of prophylactic vertebroplasty on the incidence of proximal junctional complications after posterior spinal fusion to the low thoracic spine. Spine Deform 2013;1(2):132-8.

[44] Panjabi MM. The stabilizing system of the spine. Part II. Neutral zone and instability hypothesis. J Spinal Disord 1992;5(4):390-6. discussion 7. 